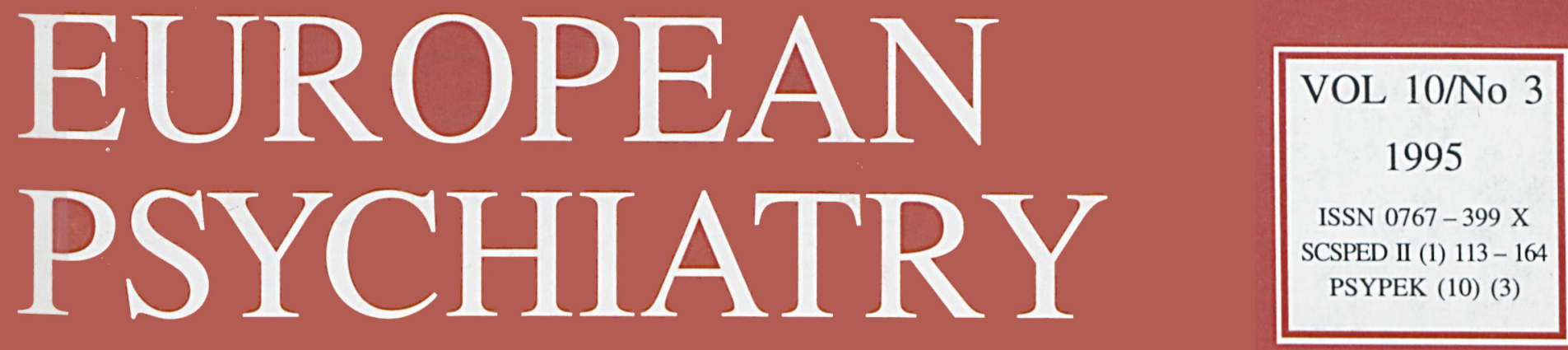

THE JOURNAL OF THE ASSOCIATION OF EUROPEAN PSYCHIATRISTS

\title{
GENERAL REVIEWS
}

Case management: a critical review of the outcome literature

F Holloway, N Oliver, E Collins, J Carson (London, UK)

Decreasing rates of incident schizophrenia cases in psychiatric service: a review of the literature

P Munk-Jørgensen (Risskov, Denmark)

\section{ORIGINAL ARTICLES}

Antidepressant effect of 5-methoxypsoralen: the melatonin synchronizer hy,

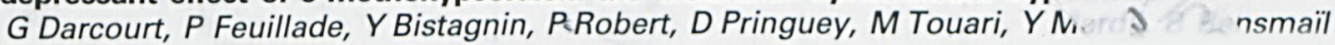
(Nice, France; Constantine, Algeria)

Clinical and socio-demographic correlates of coping strategies in relatives of s sizconic patients

L Magliano, F Veltro, M Guarneri, C Marasco (Naples, Italy)

\section{SHORT COMMUNICATION}

Pharmacotherapeutic response of DST-positive borderline patients

VP Kontaxakis, GN Christodoulou, M Markianos, C Stefanis (Athens, Greece)

\section{CASE REPORT}

Synergistic effect of a clozapine-nimodipine association

A Lenzi, A Rotondo, D Marazziti (Pisa, Italy)

\section{LETTERS TO THE EDITOR}

Toxic epidermal necrolysis after paroxetine treatment

$P$ Wolkenstein, D Cremniter, JC Roujeau (Créteil, France)

Metoclopramide-induced panic attacks

H Chabrol, JL Montastruc (Toulouse, France)

List of Reviewers

Agenda

Instructions to authors

inside back cover

Cited/abstracted in: Biological Abstracts/Biosis, CNRS/Pascal, Current Contents/Clinical Medicine and Social and Behavioral Sciences, Excerpta Medica/EMbase, Psychological Abstracts

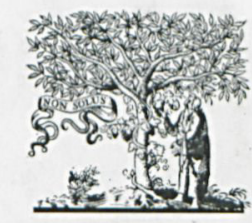




\section{EUROPEAN PSYCHIATRY}

\section{THE JOURNAL OF THE ASSOCIATION OF EUROPEAN PSYCHIATRISTS}

Editors: C Ballus (Barcelona), JD Guelfi (Paris), H Heimann (Tübingen), R Murray (London) Co-Editors: M Maj (Naples), CB Pull (Luxembourg), M Ackenheil (Munich), P Bech (Copenhagen)

Deputy editors: P Boyer, Y Lecrubier (Paris). Secretariat: Hôpital de la Salpêtrière, Pavillon Clérambault, 47, bd de l'Hôpital, 75651 Paris Cedex 13, France. Tel: 33 (1) 42161656 . Fax: 33 (1) 45852800

Advisory board

J Adès, Colombes, France

HS Akiskal, Rockville, MD, USA

NC Andreasen, Iowa City, IA, USA

J Angst, Zurich, Switzerland

M Ansseau, Liège, Belgium

P Baumann, Lausanne, Switzerland

H Beckmann, Würzburg, Germany

P Berner, Vienna, Austria

A Bertelsen, Risskov, Denmark

J Bibert, Cadiz, Spain

J Birley, London, UK

JC Bisserbe, Meudon, France

B Bondy, Munich, Germany

JP Boulenger, Sherbrooke, Canada

M Bourgeois, Bordeaux, France

F Brambilla, Milan, Italy

I Brockington, Birmingham, UK

A Clare, Dublin, Ireland

F Clerget-Darpoux, Paris, France

$\checkmark$ Conde Lopez, Valladolid, Spain

S Consoli, Paris, France

P Cosyns, Antwerp, Belgium

J Cottraux, Lyon, France

$M$ von Cranach, Kaufbeuren, Germany

A Dahl, Oslo, Norway

JM Danion, Strasbourg, France

JFW Deakin, Manchester, UK

$\mathrm{M}$ de Bonis, Le Kremlin Bicêtre, France

$\mathrm{H}$ Dufour, Lausanne, Switzerland

R Engel, Munich, Germany

T Fahy, Galway, Ireland

L Farde, Stockholm. Sweden

A Féline, Le Kremlin Bicêtre, France

A Fernandes da Fonseca, Porto, Portugal

F Ferrero, Geneva, Switzerland

M Fichter, Prien am Chiemsee, Germany

$\mathrm{H}$ Freeman, London, $U K$

HJ Gaertner, Tübingen, Germany

D Goldberg, Manchester, UK

I Hand, Hamburg, Germany

H Häfner, Mannheim, Germany

T Helgason, Reykjavik, Iceland

H Hippius, Munich, Germany

A Jablenski, Sofia, Bulgaria

E Johnston, Edinburgh, UK

S Kasper, Vienna, Austria

M Kastrup, Hvidovre, Denmark

D Kemali, Naples, Italy

R Kendell, Edinburgh, UK

D Klein, New York, NY, USA

R Klein, New York, NY, USA

S Langer, Paris, France

J Lellouch, Villejuif, France

P Lemoine, Lyon, Paris

T Lemperière, Colombes, France

JP Lépine, Paris, France

OM Lesch, Vienna, Austria

SW Lewis, London, $U K$

H Lôo, Paris, France

JJ Lopez-Ibor, Madrid, Spain

P McGuffin, Cardiff, UK

W Maier, Mainz, Germany

A Mann, London, $U K$

K Mann, Tübingen, Germany

I Marks, London, UK

J Marlet, Venray, The Netherlands

J Massanna Ronquillo, Barcelona, Spain

J Mendlewicz, Brussels, Belgium

HJ Möller, Munich, Germany

N Müller, Munich, Germany
M Musalek, Vienna, Austria

D Naber, Munich, Germany

E O'Callaghan, Dublin, Ireland

Y Ono, Tokyo, Japan

M Patris, Strasbourg, France

J Pellet, Saint-Etienne, France

C Perris, Umeä, Sweden

P Pichot, Paris, France

T Pohlmächer, Munich, Germany

H Pope, Belmont, MA, USA

AJ Puech, Paris, France

G Racagni, Milan, Italy

N Retterst $ø 1$, Oslo, Nonway

M A Ron, London, $U K$

R Rosenberg, Risskov, Denmark

$\mathrm{M}$ Roth, Cambridge, UK

F Rouillon, Colombes, France

J Saiz-Ruiz, Madrid, Spain

A Sanchez-Blanque, Zaragoza, Spain

N Sartorius, Geneva, Switzerland

F Schulsinger, Copenhagen, Denmark

G Sedvall, Stockholm, Sweden

L Singer, Strasbourg, France

CN Stefanis, Athens, Greece

E Straube, Tübingen, Germany

E Taylor, London, $U K$

$P$ Taylor, London, $U K$

L Träskman-Bendz, Lund, Sweden

J Vallejo Ruibola, Barcelona, Spain

L Waintraub, Paris, France

D Widlöcher, Paris, France

J Wilmotte, Marchienne du Pont, Belgium

J Wing, London, $U K$

FT Zimmer, Tübingen, Germany

J Zohar, Beer-Sheva, Israel

\section{Association of European Psychiatrists}

President: R Murray (London); Past President: P Bech (Copenhagen); President Elect: J Angst (Zürich); Secretary General: CB Pull (Luxembourg); Treasurer: M Patris (Strasbourg); Counsellors: H Höfner (Mannheim), Y Lecrubier (Paris); Section: L Singer (Strasbourg).

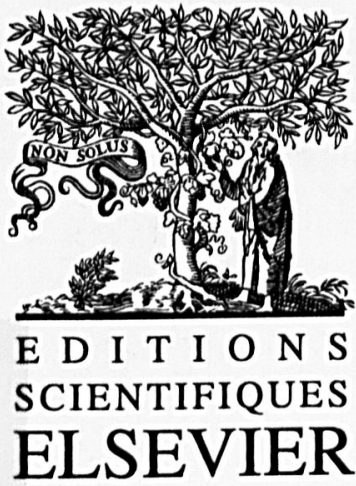

141, rue de Javel 75747 Paris Cedex 15, France

QA member of the Reed Elsevier Medical group
MEDICAL DEPARTMENT

Tel: 33 (1) 45589026 - Fax: 33 (1) 45589422

Desk editor - Valerie-Ann Libert: 33 (1) 45589031

Advertising - Anne Blanvillain: 33 (1) 45589024

Consulting translator: P Rousseau-Cunningham

\section{SUBSCRIPTIONS - Tel: 33 (1) 45589067 - Fax: 33 (1) 45589424}

1995 (Vol 10) Annual subscription; 8 issues:

- France: $1780 \mathrm{FF} \bullet$ European Union (with VAT registration no) and rest of the world: $1995 \mathrm{FF} \bullet$ European Union (individuals): $2037 \mathrm{FF} \bullet$ North, Central and South America: 294 US \$• Students (copy of the student card requested) France: $828 \mathrm{FF}$; rest of the world: $1222 \mathrm{FF}$.

Including air delivery.

Address order and payment to: Éditions scientifiques Elsevier

- by check or credit card (CB, MasterCard or Visa; indicate no and expiration date)

- by transfer: CCP Paris no 30041000011904540 H 020/70.

- Subscriptions begin 4 weeks after receipt of payment and start with the first issue of the calendar year.

- Back issues and volumes are a\%ailable from the publisher.

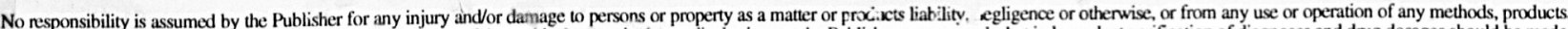

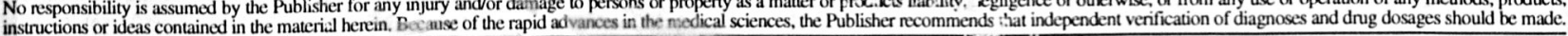

(C) 1995 fititions scientificues Elsev.... Par.

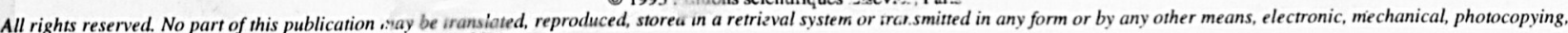
recording or otherwise, without prior permission of the publisher. 